\title{
La gestión administrativa y compromiso organizacional en instituciones educativas
}

\section{Administrative management and organizational commitment in educational institutions}

\author{
Vanessa Anchelia-Gonzales \\ Universidad Nacional Mayor de San Marcos, Perú \\ Orcid: https://orcid.org/0000-0002-3546-7298 \\ Miguel Inga-Arias \\ Universidad Nacional Mayor de San Marcos, Perú \\ Orcid: https://orcid.org/0000-0002-1588-0181 \\ Pablo Olivares-Rodríguez \\ Universidad Nacional Federico Villareal, Perú \\ Orcid: https://orcid.org/0000-0002-2303-3986 \\ Jorge Luis Escalante-Flores \\ Universidad de Lima, Perú \\ Orcid: https://orcid.org/0000-0002-4942-5283
}

*Correspondence

Email: drasoe2210@gmail.com
Cite as:

\footnotetext{
Anchelia-Gonzales, V., Inga-Arias, M., Olivares-Rodríguez, Escalante-Flores, J. (2021). La gestión administrativa y compromiso organizacional en instituciones educativas. Propósitos y Representaciones, 9 (SPE1), e899. Doi: http://dx.doi.org/10.20511/pyr2021.v9nSPE1.899
} 


\section{Resumen}

El presente estudio se enfocó en determinar la relación de la gestión administrativa con el compromiso organizacional en trabajadores del sector educación. Se ha desarrollado según el paradigma positivista de enfoque cuantitativo, investigación básica, diseño no experimental, corte transversal, tipo correlacional, aplicando el método hipotético deductivo. La población estuvo conformada por 88 trabajadores del sector educación tales como docentes, personal administrativo y directivos. Se emplearon dos cuestionarios uno de gestión administrativa y otro de Compromiso organizacional, los que fueron sometidos a criterios de expertos; dichos instrumentos obtuvieron 0.885 y 0.801 de fiabilidad por alfa de Cronbach. La evaluación de los resultados fue hecha por Rho de Spearman, obteniéndose un valor de para Gestión administrativa y de para Compromiso organizacional. Se concluyó que existió una relación positiva y significativa entre ambas variables, estableciéndose que mientras exista una buena gestión, el compromiso de los trabajadores se incrementa progresivamente.

Palabras clave: gestión administrativa, compromiso organizacional, compromiso afectivo, compromiso de continuidad.

\section{Summary}

The present study focused on determining the relationship between administrative management and organizational commitment in workers in the education sector. It has been developed according to the positivist paradigm of quantitative approach, basic research, non-experimental design, cross-sectional, correlational type, applying the hypothetical deductive method. The population was made up of 88 workers from the education sector such as teachers, administrative personnel and managers. Two questionnaires were used, one for administrative management and the other for Organizational Commitment, which were subjected to expert criteria; These instruments obtained 0.885 and 0.801 reliability by Cronbach's alpha. The evaluation of the results was made by Rho de Spearman, obtaining a value of for Administrative management and of for Organizational commitment. It was concluded that there was a positive and significant relationship between both variables, establishing that as long as there is good management, the commitment of the workers increases progressively.

Keywords: administrative management, organizational commitment, affective commitment, continuity commitment

\section{Introducción}

Se ha generado una atención creciente, de forma especial en las últimas décadas, al aspecto etológico de los individuos en el rubro laboral, ya que, según Fonseca, Cruz \& Chacón (2019) se ha dado un especial reconocimiento a los empleados y al entorno donde se desenvuelven como un hecho trascendente que repercute sobre el desarrollo y progreso de una organización. Sin embargo, gran parte de la investigación que estudió las relaciones entre la satisfacción laboral, el compromiso organizacional y las actitudes hacia el cambio organizacional y sus dimensiones se ha llevado a cabo en entornos culturales, ya sean orientales u occidentales, que difieren en muchos aspectos a los que se tiene en los Emiratos Árabes (Yousef, 2017).

Los investigadores Peña, Díaz, Chávez \& Sánchez (2016) mencionaron que si una institución posee una adecuada funcionalidad es porque ha implementado actividades innovadoras generadas por los empleados, las cuales son un plus frente al marco de responsabilidades propias de un puesto dado, pero que al mismo tiempo es una evidencia clara del nivel de identificación con la misión y visión organizacional. 
De acuerdo con Calderón (2016) el conjunto de ideas, creencias, normas, tradiciones y costumbres que los constituyentes de una determinada organización han generado y que direcciona su actuación, así como el nivel de productividad y la satisfacción laboral, se conoce como cultura organizacional.

Al respecto se tiene el trabajo de Pacheco, Robles \& Ospino (2018) sobre el análisis de la gestión administrativa en 25 instituciones educativas rurales en Santa Marta, Colombia, para lo cual asumieron una investigación descriptiva, de campo y transversal, empleando un instrumento de 48 reactivos con respuestas tipo Likert; siendo sus resultados la inexistencia de enfoque administrativo predominante en las instituciones analizadas, añadiendo además que un $72 \%$ de los directivos no emplea soportes de decisión en su proceso de gestión administrativa.

Por otro lado, cabe mencionar el estudio realizado por Fonseca et al. (2019) que se orientó a sostener la validez de constructo, criterio y consistencia interna de un instrumento que midió el nivel de compromiso organizacional; para lo cual se valieron del proceso de redes semánticas naturales modificadas con la finalidad de generar una base de reactivos, todos validados, en base del cual se generó un instrumento conformado por 40 reactivos que se aplicaron a 140 trabajadores en Guanajuato, México; dando como resultado la identificación de una serie de factores con un valor de alfa de 0.86 a 0.89 los que se consideraron como categóricamente congruentes.

Por su parte Berberoglu (2018) desarrolló un estudio con el objetivo de evaluar las percepciones de los empleados de la salud sobre el clima organizacional y probar el impacto hipotético del clima organizacional en el compromiso organizacional y el desempeño organizacional percibido; para lo cual adoptó un enfoque cuantitativo, mediante la recopilación de datos de los trabajadores de la salud actualmente empleados en hospitales públicos en el norte de Chipre, utilizando un cuestionario autoadministrado. Los datos recopilados se analizaron con la ayuda del paquete estadístico para ciencias sociales, y se utilizaron análisis de regresión lineal y ANOVA para probar la hipótesis. Los resultados revelaron que el clima organizacional está altamente correlacionado con el compromiso organizacional y el desempeño organizacional percibido. Los resultados de la regresión lineal simple indicaron que el clima organizacional es significativo para predecir el compromiso organizacional y el desempeño organizacional percibido. De lo anterior concluyó que existió una relación positiva y lineal entre el clima organizacional con el compromiso organizacional y el desempeño organizacional percibido, ya que los resultados del análisis de regresión sugirieron que el clima organizacional tiene un impacto en la predicción del compromiso organizacional y la percepción organizacional.

Un estudio empírico, que incluyó a 566 personas, empleadas por 8 empresas, destinado a identificar y cuantificar la relación entre las características laborales, el compromiso organizacional, la satisfacción laboral, la participación laboral y las políticas y procedimientos organizacionales en la economía en transición de Serbia, sudeste de Europa, fuer el que realizaron Ćulibrk, Delić Mitrović \& Ćulibrk (2018). Dicho estudio reveló que los modelos existentes de motivación laboral deben adaptarse para ajustarse a los datos empíricos, lo que resultó en un modelo de investigación revisado elaborado en el documento. En el modelo propuesto, la participación en el trabajo media parcialmente el efecto de la satisfacción laboral en el compromiso organizacional. La satisfacción laboral en Serbia se ve afectada por las características laborales, pero a diferencia de muchos estudios realizados en economías desarrolladas, las políticas y procedimientos organizativos no parecen afectar significativamente la satisfacción de los empleados.

También cabe destacar el estudio realizado por Yousef (2017), quien se avocó a investigar las relaciones directas e indirectas entre la satisfacción laboral, el compromiso 
organizacional y las actitudes hacia el cambio organizacional y sus dimensiones. Los resultados obtenidos indicaron que los empleados de los departamentos investigados están muy satisfechos con la supervisión y los compañeros de trabajo, mientras que están ligeramente satisfechos con las condiciones laborales y la seguridad laboral, pero tienen una baja satisfacción con las facetas de remuneración y promoción del puesto. Así mismo dichos resultados demostraron además que los empleados de los departamentos investigados permanecen en sus departamentos actuales, ya sea porque quieren hacerlo o porque tienen que hacerlo, pero no porque sientan que deben hacerlo.

Por otro lado, se tiene el trabajo desarrollado por Peña et al. (2016) que se orientaron a identificar cuáles fueron los factores de mayor relevancia del compromiso organizacional del rubro de pequeñas empresas en un sector de México; para lo cual estimaron una muestra formada por 60 trabajadores, empleando para la recolección de data el un instrumento de Meyer y Allen (1991), conformado por 21 reactivos con respuestas tipo Likert el cual permite diagnosticar los compromisos de tipo organizacional, afectivo, de continuidad y normativo. Se concluyó que hubo una correlación altamente significativa entre el compromiso afectivo y de continuidad con el compromiso normativo, y la única asociación moderada se da entre el compromiso afectivo y el compromiso de continuidad.

El trabajo descriptivo-correlacional desarrollado por Osorio, Ramos \& Walteros (2016) se orientó a establecer el vínculo entre los valores personales y el compromiso organizacional de 128 funcionarios de una institución de educación superior de Bogotá, siendo la colecta de data basada en cuestionarios adaptados lingüística, semántica y culturalmente, denominados cuestionario de Retratos de Schwartz y el cuestionario de compromiso organizacional de Meyer. Aplicaron el modelo de ecuaciones estructurales para demostrar un porcentaje bajo de la varianza de los datos. Obtuvieron un modelo con tres variables emergentes. Concluyeron que la relación entre los constructos propuestos es compleja e involucra muchas más variables, siendo más apropiado si se incluye un análisis de la coherencia de los valores declarados y vivenciados organizacionalmente y los valores de los individuos en función del compromiso organizacional.

La calidad de los funcionarios de una organización, sus conocimientos y habilidades, su entusiasmo y satisfacción en sus cargos, y su iniciativa para generar riqueza repercuten con fuerza en la productividad, el nivel de servicio al cliente, la reputación y la competitividad de la organización.

En razón a que los recursos humanos constituyen el elemento esencial en cada componente de la organización, su administración eficaz se fundamenta en la responsabilidad de cada gerente en las distintas áreas funcionales de la organización. Por tanto, cualquiera sea el área empresarial seleccionada el futuro administrador necesita fundamentar su visión de cómo tratar los asuntos relacionados con las personas y obtener una perspectiva de recursos humanos que le permita alcanzar el éxito profesional y liderar su organización hacia la excelencia y competitividad. Por lo anteriormente mencionado el presente trabajo se planteó como objetivo primordial determinar la relación de la gestión administrativa y el compromiso organizacional y como objetivos accesorios: establecer la relación de la gestión administrativa frente a los compromisos afectivo y de continuidad al interior de una institución educativa.

\section{Gestión administrativa}

De acuerdo con Arnoletto (2014) la gestión administrativa se define como el conjunto de actividades y procesos definidos que el o los directivos tienden a desarrollar en función de los recursos que tengan a disposición como por ejemplo recursos materiales, humanos, entre otros. Según lo manifestado por Johnes (2015) en cada institución el proceso de gestión administrativa es función directa del consejo directivo ya que según dicho autor el consejo académico no ejerce 
función administrativa alguna.

Por su parte Ocando (2017) mencionó que la gestión administrativa equivale al proceso o mecanismo desarrollado por un único o diversos responsables cuya finalidad es direccionar el desempeño de los trabajadores, lo que se traduce en qué tan adecuado es el proceso de empleo de recursos de los que disponen. Así mismo Castro (2016) mencionaron que el proceso de gestión marca la pauta que orienta el desarrollo socioeconómico y que al mismo tiempo garantiza el empleo optimizado de todos los recursos disponibles orientando el desempeño de los trabajadores hacia la consolidación de los fines trazados por la organización y sus directivos.

La gestión administrativa, según Junshan (2017), deben ser entendidas como una serie de operaciones que deben ser ejecutadas con la finalidad de movilizar una serie de recursos que se tengan a disposición (bienes, servicios, personal, entre otros) a fin de plasmar los objetivos de una institución; razón por la cual dicho proceso compete una serie de requerimientos tales como planificación, asignación de procesos, delimitación de funciones y en especial la toma de decisiones y manejo de conflictos que pudiesen ocurrir en el contexto de la organización. De lo anterior descrito se resume que, para poder administrar una gestión educativa, es requisito primordial tener presente todos los elementos que forman parte de la dinámica institucional, desde la relación del centro con su medio, asignación de tareas y la organización para la enseñanza, hasta el manejo del personal y las demandas administrativas del plantel.

Según Cárdenas, Farías \& Méndez (2017) el proceso administrativo está conformado por una serie de componentes ordenados a manera de un sistema organizado estructuralmente que canaliza los esfuerzos de forma cooperativa de todos los componentes, no de manera individual, a fin de consolidar objetivos comunes. De lo expuesto, se podría considerar que administrar un centro educativo requiere del esfuerzo coordinado, trabajo en equipo, de mecanismos de adaptación a los cambios sucedáneos que pudiesen darse en el sistema educativo, todo ello con el fin de conseguir resultados que posibiliten y sostengan la competitividad en el medio. De dicha manera, Ocando (2017) se refirió a la labor del director como el administrador que ejerce un estamento de poder lo que le garantiza generar, organizar y coordinar las actividades, tomar decisiones, planificación y desarrollo de proyectos; todo ello sin dejar de tener presente el norte de mantener el orden y control institucional, así como avocarse en el desarrollo continuo de su espectro de acciones y mantener una proactividad continua.

Por su parte Altinay, Altinay, Dagli \& Cifci (2018) mencionaron que administrar se relaciona con el desenvolvimiento del personal idóneo que gravita en función de los objetivos institucionales, manejando operativamente los recurso que tenga a disposición, asumiendo la toma de decisiones y que de forma especial el administrador del área educativa asume las disposiciones del currículo, planes de estudio, en función de la gestión escolar, siempre con la capacidad de poder identificar las necesidades y requerimientos, así como la delimitación de rangos y funciones de los subordinados, cumpliendo con los fines y objetivos propuestos, todo ello en función de los recursos que tenga a su disposición, teniendo para ello la capacidad de liderazgo, empatía, comunicación, asertividad y de forma especial de motivación de su personal incentivando continuamente a la consolidación de los objetivos de la institución.

Los candidatos que se espera participen en el campo de la administración educativa deben estar capacitados tanto en el servicio como en el proceso de desarrollo continuo. El conocimiento y la formación docente son necesarios, pero no suficientes para la gestión (Altinay et al., 2018). En otras palabras, la visión de que los maestros exitosos serán administradores escolares exitosos está lejos de ser científica. Porque tanto la docencia como la gestión son profesiones y especializaciones independientes. En términos de habilidades gerenciales y de 
personal docente, los administradores escolares deben crecer en servicio y mejorar constantemente (Askal, 2015).

Administrar en el sector educación, es una actividad consiente ligada a la acción de gestionar, de carácter público o privado, que urge, ergo, de un mecanismo que haga factible la planificación, asignación de suministros y funciones, el adecuado flujo de información para la de decisiones en la implementación de acciones que permita la eficiencia institucional. Desde otro aspecto, el gestor educativo debe ser consiente que la interacción de sus trabajadores será afectada por el nivel cultural (creencias, valores, modos de interpretar y percibir la realidad y las normas, actitudes, formas de expresión). Del mismo modo es prioritario percibir a las instituciones educativas como organizaciones que se hallan en constante proceso de aprendizaje, deseosos de conocimientos, forjando competitividad; estos procesos facilitaran los procesos administrativos.

\section{Compromiso organizacional}

De forma retrospectiva, cabe citar que la idea más estructurada sobre compromiso organizacional fue dada por Meyer y Allen quienes adoptaron un punto de vista diferente y conceptualizaron el modelo de tres componentes del compromiso organizacional (Fonseca et al., 2019; Berberoglu, 2018, Yousef, 2017). Al respecto los mencionados Allen y Meyer (1991) explicaron los tres componentes del compromiso organizacional tales como compromiso "Afectivo", "Continuidad" y "Normativo". En sus conceptualizaciones, los empleados con niveles más altos de compromiso afectivo permanecen en sus organizaciones porque "quieren", aquellos con altos niveles de compromiso de continuidad permanecen en sus organizaciones porque "necesitan" y aquellos asociados con el compromiso normativo permanecen porque sienten que "debería" hacerlo. Según la definición de Meyer y Allen (1991), el concepto de compromiso organizacional es un estado psicológico que caracteriza la relación del empleado con la organización y tiene implicaciones para la decisión de continuar o dejar de ser miembro de la organización.

Cualquier organización logra el éxito por medio del compromiso organizacional de su capital humano, el cual es un importante activo que sobre el cual actúa la dirección de una empresa para evaluar la lealtad y el nivel de vinculación de los empleados con la organización, por ello Peña et al. (2016) lo consideran como trascendente que las empresas conozcan el nivel de compromiso de sus miembros. Según Ćulibrk et al. (2018) se entiende por compromiso organizacional al grado en que los empleados se identifican con la organización en la que trabajan, qué tan comprometidos están con la organización y si están listos para dejarla. Por otro lado, Cesário \& Chambel (2017) manifestaron que el compromiso organizacional se puede considerar como una extensión de la satisfacción laboral, ya que se trata de la actitud positiva que tiene un empleado, no hacia su propio trabajo, sino hacia la organización. Las emociones, sin embargo, son mucho más fuertes en el caso del compromiso organizacional y se caracteriza por el apego del empleado a la organización y la disposición a hacer sacrificios por la organización (Valaei \& Rezaei, 2016).

Por su parte Chordiya, Sabharwal \& Goodman (2017) manifestaron que una organización será sostenible, siempre que el compromiso asumido se encamine de las instancias superiores hacia abajo y, que además posea un continuo proceso de seguimiento por parte de la alta dirección de la institución; por lo que se asume que el compromiso organizacional se encuentra promovido desde las instancias jerárquicas más elevadas.

Por su parte Yousef (2017) mencionó que la satisfacción laboral, el compromiso organizacional y las actitudes hacia el cambio organizacional y las relaciones entre estos constructos han sido tema de numerosos artículos en las últimas décadas. Esto se debe 
principalmente a la importancia de estos constructos para empleados y empleadores. Sobre el mismo punto hay que considerar lo manifestado por Valaei \& Rezaei (2016) quienes consideraron el hecho de que existe un conflicto con respecto a la relación causal entre la satisfacción laboral y el compromiso organizacional, siendo obvio que ambos aspectos conectan y vinculan variables que impactan los resultados organizacionales, por lo que además agregaron que diversos investigadores han establecido que la satisfacción laboral antecede causalmente al compromiso organizacional.

Según Calderón (2016) en la actualidad se han desarrollado muchos estudios que ponen de relieve la importancia de aspectos relacionados con el compromiso organizacional de los miembros de una determinada organización y su vinculación con las mejoras significativa en el bienestar laboral, así como la elevación de la productividad de los mismos. Por su parte Fonseca et al. (2019) estimaron que el compromiso organizacional se encuentra estrechamente relacionado con la satisfacción laboral y ello repercute en el nivel de productividad de los individuos.

Según lo referenciado por Calderón, Laca, Pando y Pedroza (2015) acerca de estudios sobre el compromiso organizacional, los mismos han evidenciado que hay pruebas que denotan que el compromiso afectivo tiende a generar atenuantes sobre sentimientos de competencias entre los trabajadores y la mejora sustancial de la autoestima. En la misma línea se encuentran Osorio et al. (2016) que establecieron que el compromiso afectivo se traduce como factor determinante que se manifiesta en la dedicación y la lealtad de los trabajadores, aspecto que coadyuva al desarrollo del sentido de pertenencia e implicación en las actividades de la organización.

De acuerdo con Berberoglu (2018) conceptos como la satisfacción laboral, la necesidad de logro, la afiliación y el poder, la eficacia y el desempeño organizacionales generales y el compromiso organizacional son consecuencias del clima organizacional percibido. Según el citado autor el compromiso organizacional de los empleados con su organización tiene una relación significativa e influencia en el desempeño organizacional general, así como el desempeño individual de los empleados también se correlaciona con el compromiso organizacional.

En la revisión de la literatura sobre los antecedentes y las consecuencias del compromiso organizacional, los antecedentes más encontrados son las características personales, la estructura organizacional, la permanencia, las recompensas, la capacitación y los valores laborales, mientras que las consecuencias son principalmente un mayor rendimiento, motivación y menor rotación de los empleados. Intenciones (Chordiya, Sabharwal \& Goodman, 2017). Según lo manifestado por Berberoglu (2018) las características demográficas como el estado civil de los trabajadores y el nivel de compromiso afectivo y compromiso normativo se encuentran positivas y, por lo tanto, se encuentra que existe una diferencia estadísticamente significativa en los niveles de compromiso normativo con respecto al estado educativo de los estudios revisados por el citado autor.

\section{Metodología}

La presente investigación se enmarco en el paradigma positivista, con enfoque cuantitativo, siendo el tipo de estudio el básico, de diseño no experimental, corte transversal, correlacional. Se trabajó con el método hipotético deductivo. La población fue de tipo censal ya que se trabajó con la totalidad de los componentes del cuadro administrativo de la institución educativa, siendo un total de 88 individuos los mismos que estuvieron comprendidos entre docentes de niveles primaria y secundaria, auxiliares, así como personal administrativo y logístico. 
Como instrumento para recopilar datos se han confeccionado dos cuestionarios, siendo el primero denominado 'Cuestionario para evaluar la Gestión administrativa' el cual estuvo conformado por 24 reactivos; mientras que el segundo se denominó 'Cuestionario de Compromiso organizacional' conformado por 18 reactivos los cuales fueron sometidos a la validación de cinco expertos en el rubro y el análisis de confiabilidad se muestra en la tabla 1.

Tabla 1.

Fiabilidad de los instrumentos

\begin{tabular}{lcc}
\hline variable & Alfa de Cronbach & N de elementos \\
\hline Gestión administrativa & 0.885 & 24 \\
Compromiso organizacional & 0.801 & 18 \\
\hline
\end{tabular}

Los datos obtenidos de las encuestas recabadas se vaciaron en plantillas del programa Microsoft Excel (Office 2016) y el procesamiento estadístico descriptivo como analítico fue hecho con el programa SPSS versión 24 de IBM con la finalidad de corroborar las hipótesis planteadas y generar los gráficos y tablas correspondientes.

\section{Resultados}

El examen de relación entre la gestión administrativa y el compromiso organizacional se muestra en la tabla 2. De acuerdo con la evaluación de los resultados obtenidos de las encuestas respectivas, se tuvo que la evaluación del coeficiente de correlación de rho de Spearman generó un valor de 0.361 con un nivel de significancia menor a 0.05 lo que se tradujo en que el nexo entre gestión administrativa y compromiso organizacional fue positivo, pero bajo en el personal encuestado.

Tabla 2.

Coeficiente de correlación entre la gestión administrativa y el compromiso organizacional.

\begin{tabular}{ccc}
\hline & & Compromiso organizacional \\
\hline \multirow{2}{*}{ Gestión } & Correlación de Spearman & $.361^{*}$ \\
administrativa & Sig. $(\mathrm{p})$ & .001 \\
& $\mathrm{~N}$ & 88 \\
\hline
\end{tabular}

Por otro lado, la imagen 1 muestra que el $56 \%$ de los trabajadores que calificaron como buena la gestión administrativa de la institución, a su vez presentaron un nivel alto de compromiso organizacional, mientras que el $20 \%$ de los trabajadores que califican como buena la gestión administrativa de su institución a su vez presentan un nivel moderado de compromiso organizacional y el $24 \%$ de los mismos lo asumió como regular dicho vínculo. 


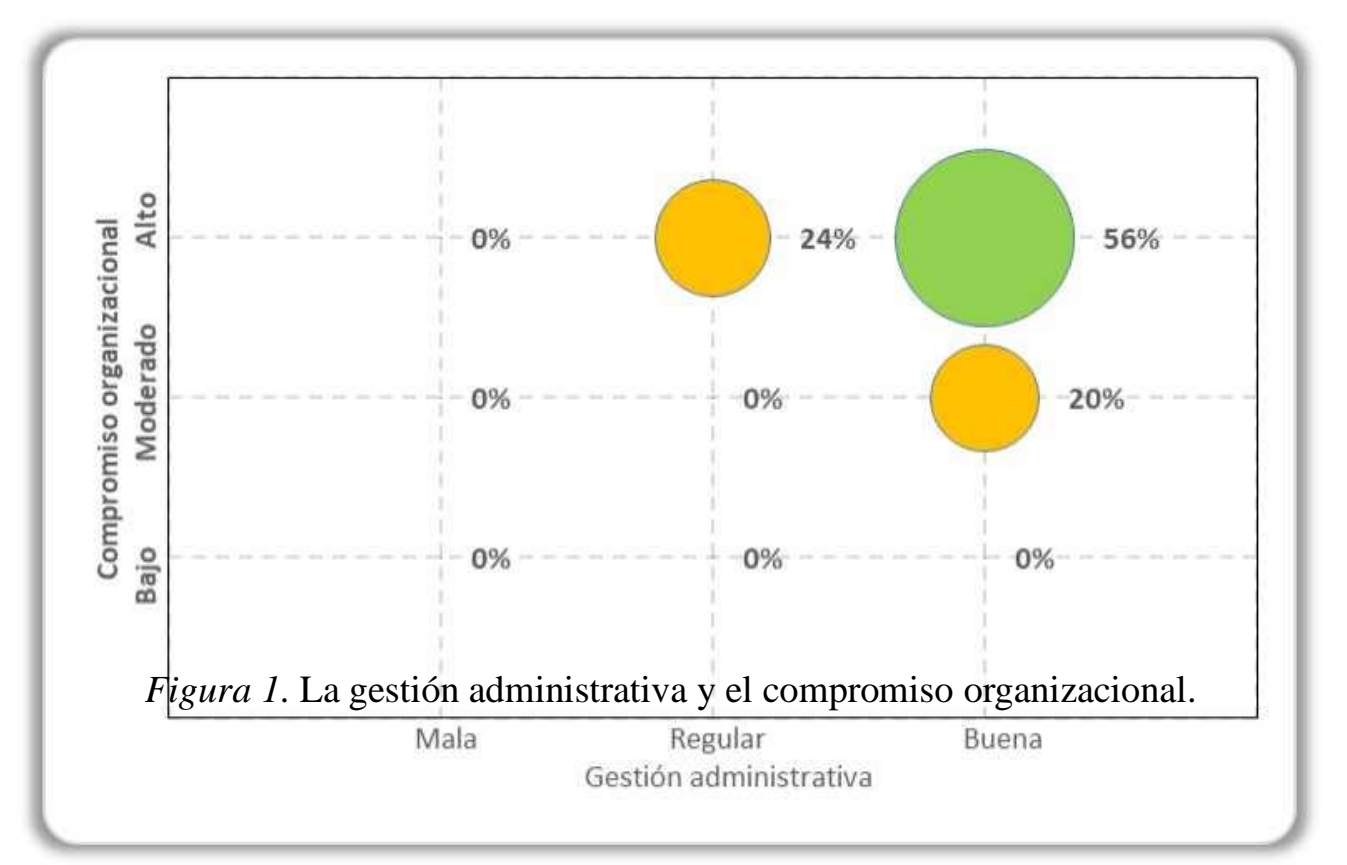

La relación que existió entre la dimensión compromiso afectivo frente a la gestión administrativa se muestra en la tabla 3. De la misma se rescata que el valor del coeficiente de Spearman fue de 0.604 con un nivel de significancia menor a 0.000 , hecho que se tradujo en que si hubo una relación positiva y moderada entre los parámetros señalados al interior de los trabajadores de la institución.

Tabla 3.

Coeficiente de correlación entre la gestión administrativa y el compromiso afectivo.

\begin{tabular}{ccc}
\hline & & Compromiso afectivo \\
\hline \multirow{2}{*}{ Gestión } & Correlación de Spearman & $.604^{* *}$ \\
administrativa & Sig. (p) & .000 \\
& $\mathrm{~N}$ & 88 \\
\hline
\end{tabular}

Por otro lado, la figura 2 muestra que el 74\% de los trabajadores que identificaron como 'buena' la gestión administrativa de la institución a su vez presentaron un nivel 'alto' de compromiso afectivo, mientras que solo el $2 \%$ de los mismos calificaron como 'buena' la gestión administrativa de la institución a su vez presentan un nivel moderado de compromiso afectivo; hecho que se revierte con el $24 \%$ de los citados individuos que a pesar de mostrar un nivel 'alto' de compromiso afectivo tuvieron una percepción 'regular' para la gestión administrativa. 


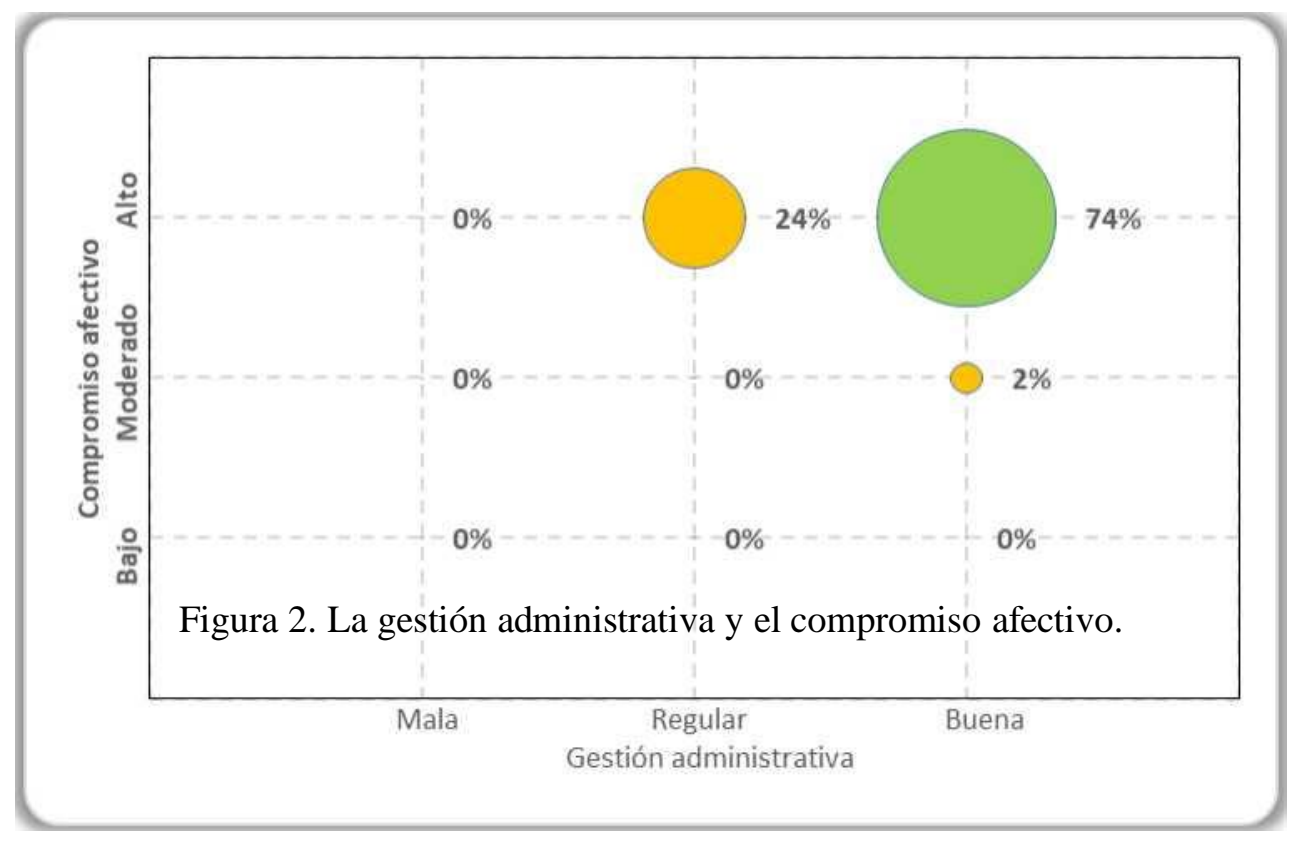

La relación que existió entre la dimensión compromiso de continuidad frente a la gestión administrativa se muestra en la tabla 4. De la misma se rescató como referente que el valor del coeficiente de Spearman fue de 0.407 con un nivel de significancia menor a 0.000 , hecho que se tradujo en que si hubo una relación positiva y moderada entre los parámetros señalados en la percepción de los trabajadores encuestados.

Tabla 4.

Coeficiente de correlación entre la gestión administrativa y el compromiso de continuidad.

\begin{tabular}{ccc}
\hline & & Compromiso de continuidad \\
\hline \multirow{2}{*}{ Gestión } & Correlación de Spearman & $.407^{* *}$ \\
administrativa & Sig. (p) & .000 \\
& $\mathrm{~N}$ & 88 \\
\hline
\end{tabular}

También cabe mencionar que de acuerdo con la figura 3 se tuvo que el $36 \%$ de los trabajadores calificó como 'buena la gestión administrativa de su institución ý a su vez presentaron un nivel alto de compromiso de continuidad, mientras que solo el $1 \%$ de los trabajadores que califican como 'buena' la gestión administrativa de su institución, pero a su vez mostraron un nivel 'bajo' de compromiso de continuidad. Lo anterior contrastó con el 39\% de los encuestados que infirieron un nivel 'moderado' de la gestión administrativa, pero que a su vez manifestaron que el compromiso de continuidad fue asumido como 'moderado'. También cabe mencionar que el compromiso de continuidad fue 'alto' en el $24 \%$ de los trabajadores encuestados, pero su identificación con la gestión administrativa fue 'regular'. 


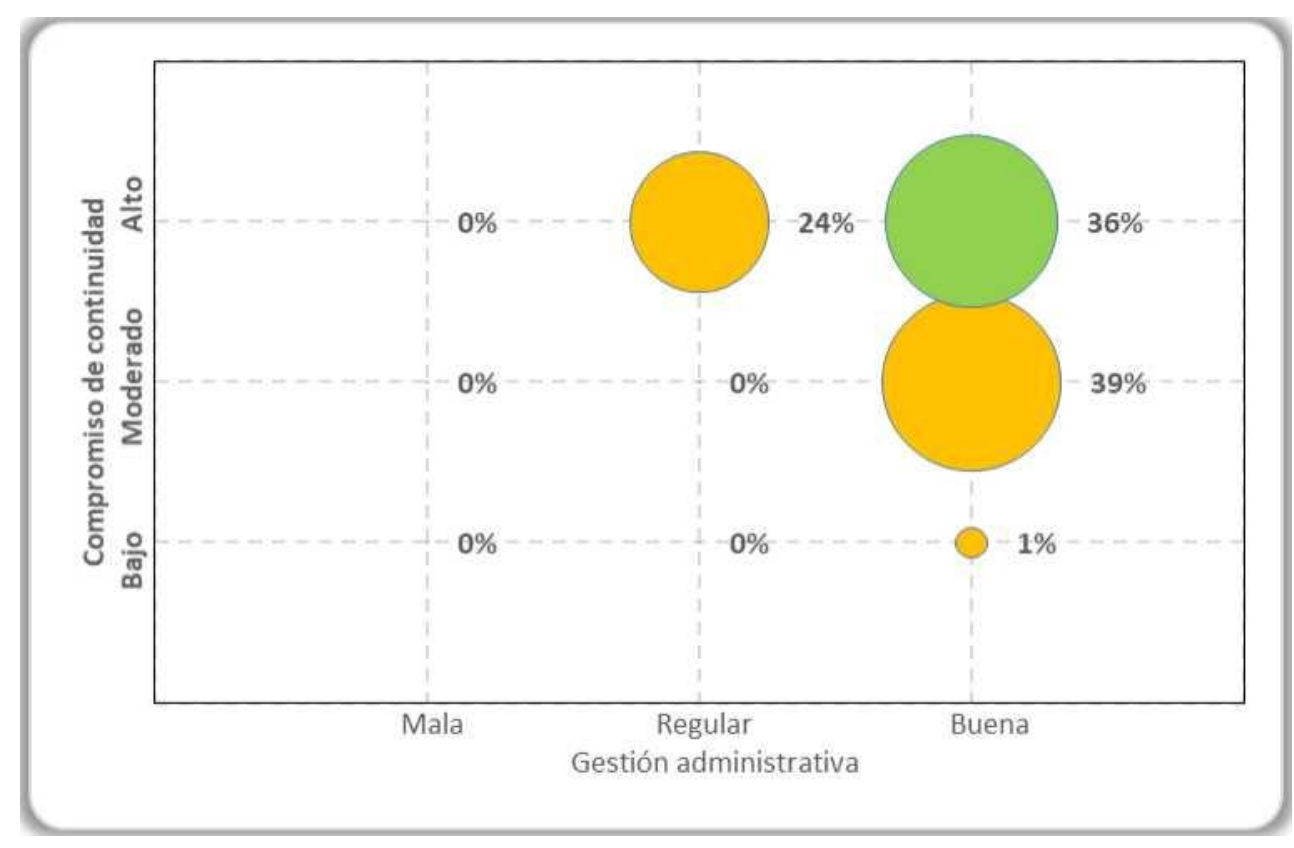

Figura 3. La gestión administrativa y el compromiso de continuidad.

\section{Discusión}

En función de los resultados obtenidos por el presente estudio, se observó que un 56\% de los trabajadores evaluados refirieron que existió una buena gestión administrativa y que a su vez su identificación con el rasgo del compromiso organizacional fue alto hecho que reluce una aceptación de los mismos respecto a los parámetros señalados. Así mismo cabe señalar que el valor del coeficiene de Spearman que arrojó un valor de 0.361 sustenta en alguna forma el hecho de lo señalado líneas arriba. Lo anterior guarda concordancia con lo referido por Aydogdu y Asikgil (2011) quienes investigaron la relación vinculante entre la satisfacción laboral, el compromiso organizacional y la intención de rotación de labores en trabajadores; revelando que la satisfacción laboral y por ende la identificación con la institución guardó una relación significativa y positiva con las tres dimensiones del compromiso organizacional (afectivo, continuo y normativo). De acuerdo con Ćulibrk et al. (2018) se tiene que la implicación laboral tiene un papel mediador entre la satisfacción laboral y el compromiso organizacional; ello se patenta en que la participación en el trabajo media la influencia de la satisfacción en el compromiso organizacional, pero esta es una mediación parcial y una gran parte del efecto de la satisfacción sobre el compromiso organizacional se logra directamente.

Del análisis de la dimensión compromiso afectivo, el $74 \%$ de los trabajadores se identificaron con una gestión administrativa como 'buena' y a su vez manifestaron que tuvieron un nivel 'alto' de compromiso afectivo, aspectos que, en cierta medida podría ser asumidos como positivos. A lo anterior hay que agregar que la correlación de los aspectos señalados arrojó un valor de 0.604 ( $\mathrm{p}$ valor < 0.05) cifra que refrenda la apreciación hallada al respecto, que puede ser traducida como una ventaja para la institución ya que el parámetro de compromiso afectivo de su personal le asegura éxito a la gestión tal como lo refirieron Peña et al. (2016) al manifestar que es un importante activo que sobre el cual actúa la dirección de una empresa para evaluar la lealtad y el nivel de vinculación de los empleados con la organización y que a su vez guarda trascendencia el hecho que una organización conozca el nivel de compromiso de sus miembros. También cabe mencionar que lo hallado le puede asegurar a los 
directivos una gestión sostenible (Chordiya et al., 2017) y que además puede asumirse que el parámetro señalado se vinculó con hechos de identificación de los trabajadores.

Para la estimación de la dimensión del compromiso de continuidad frente a la gestión administrativa, no hubo cierta constancia respecto de las estimaciones anteriores ya que se tuvo que solo el $36 \%$ de los trabajadores encuestados (un poco más de la tercera parte) se identificó con un nivel alto de compromiso de continuidad y como buena la gestión administrativa que se estuvo ejerciendo, un 39\% manifestó que su compromiso de continuidad fue moderado. Esto último puede ser sostenido en base al análisis del coeficiente de correlación del rho del Spearman que generó un valor de 0.407 que si bien es positivo, es relativamente baja, hecho que podría ser estimado en base a posibles incompatibilidades respecto del parámetro del compromiso de continuidad que presentaron los trabajadores y que si además se tiene que el $1 \%$ de los mismos estimó que los valores señalados fue bajo, es un hecho que deberá ser tenido en cuenta por los directivos a fin de poder revertir dicha tendencia. Al respecto no hay muchas precisiones, tal como lo señaló Calderón (2016, p.240) quien manifestó que "aunque han sido estudiadas las relaciones entre el bienestar laboral, la cultura de la organización y el compromiso organizacional, no se ha indagado la función de la socialización organizacional" lo que pone sobre relieve una necesidad de ahondar y precisar más trabajos al respecto. En concordancia con la postura manifestada, Arnoletto (2014) señaló que el compromiso organizacional afectivo y de continuidad son expresadas en niveles más altos en comparación del compromiso normativo, por ello se tiene que los trabajadores una institución mostrará su compromiso organizacional vinculado a su labor, de forma específica con el compromiso afectivo ya que al involucra un tema de empatía, confianza en la atención a los directivos y colegas a fin de poder cumplir los objetivos y objetivos de la institución con la cual se identifica.

Según lo planteado por Yousef (2016), se tuvo que varias dimensiones del compromiso organizacional tienen efectos directos sobre diferentes dimensiones de las actitudes hacia el cambio organizacional. También mencionó que el compromiso organizacional juega un papel mediador entre varias facetas de la satisfacción laboral y las diferentes dimensiones de las actitudes hacia el cambio organizacional.

Las estimaciones del presente estudio concuerdan con lo pedido por Yousef (2016) quien al respecto mencionó del interés de realizarse estudios longitudinales que capturen la naturaleza dinámica de la satisfacción laboral de los empleados, el compromiso organizacional y las actitudes hacia el cambio organizacional, para lograr una mayor objetividad sobre los parámetros de la eficiencia en la gestión administrativa.

\section{Referencias}

Aldana-Rivera, E. E., Tafur-Castillo, J., \& Leal-Castillo, M. (2018). Compromiso organizacional como dinámica de las instituciones del Sector Salud en BarranquillaColombia. Revista espacios, 39(11), 1-13. https://bit.ly/310b2E0

Altinay, F., Altinay, Z., Dagli, G., \& Cifci, M. (2018). Evaluation of administrative and supervisory performance of the directors of Special Education Institutions according to the teachers. Quality \& Quantity, 52(2), 1275-1286. https://doi.org/10.1007/s11135-0180699-4

Arnoletto, E. (2014). Fundamentos de la Administración de Organizaciones. Cordova Argentina: EUMED.

Aksal, F. A. (2015). Are headmasters digital leaders in school culture? Education \& 
Science/Egitim ve Bilim, 40(182), 77-86. https://bit.ly/2FxVmHU

Aydogdu, S., \& Asikgil, B. (2011). An empirical study of the relationship among job satisfaction, organizational commitment and turnover intent. International Review of Management and Marketing, 1(3), 43-53. https://bit.ly/3hbkO3V

Berberoglu, A. (2018). Impact of organizational climate on organizational commitment and perceived organizational performance: empirical evidence from public hospitals. BMC Health Services Research, 18(1), 1-9. https://doi.org/10.1186/s12913-018-3149-z

Calderón-Mafud, J. L. (2016). Socialización y compromiso organizacional: una revisión a partir del bienestar laboral. Enseñanza e investigación en Psicología, 21(3), 239-247. https://bit.ly/34chDp5

Calderón-Mafud, J.L., Laca-Arocena, F. A., Pando-Moreno, M. y Pedroza-Cabrera, F.J. (2015). Relación de la socialización organizacional y el compromiso organizacional en trabajadores mexicanos. Psicogente, 18(34), 1-11. https://bit.ly/3h8oi7u

Cárdenas-Gutiérrez, C., Farías-Martínez, G. M., \& Méndez-Castro, G. (2017). ¿Existe relación entre la gestión administrativa y la innovación educativa?: Un estudio de caso en educación superior. REICE. Revista Iberoamericana sobre Calidad, Eficacia y Cambio en Educación, 15(1), 19-35. doi: 10.15366/reice2017.15.1.002

Castro-Sánchez, M. (2016). Análisis de parámetros psicosociales, conductuales, físico deportivos y laborales de los adolescentes de Granada [tesis doctoral. Universidad de Granada. España]. Repositorio institucional https://bit.ly/3heW5fg

Cesário, F., \& Chambel, M. J. (2017). Linking organizational commitment and work engagement to employee performance. Knowledge and Process Management, 24(2), 152158. https://doi.org/10.1002/kpm.1542

Chordiya, R., Sabharwal, M., \& Goodman, D. (2017). Affective organizational commitment and job satisfaction: A cross-national comparative study. Public Administration, 95(1), 178195. https://doi.org/10.1111/padm.12306

Conley, S., \& You, S. (2017). Key influences on special education teachers' intentions to leave: The effects of administrative support and teacher team efficacy in a mediational model. Educational Management Administration \& Leadership, 45(3), 521-540. https://doi.org/10.1177/1741143215608859

Ćulibrk, J., Delić, M., Mitrović, S., \& Ćulibrk, D. (2018). Job satisfaction, organizational commitment and job involvement: The mediating role of job involvement. Frontiers in Psychology, 9, 132. https://doi.org/10.3389/fpsyg.2018.00132

Fonseca-Herrera, J. M., Cruz-Torres, C. E., \& Chacón-Gutiérrez, L. (2019). Validación del instrumento de compromiso organizacional en México: evidencias de validez de constructo, criterio y confiabilidad. Revista de Psicología, 37(1), 7-29. https://doi.org/10.18800/psico.201901.001

Johnes, J. (2015). Operational Research in education. European Journal of Operational Research, 243(3), 683-696. doi:10.1016/j.ejor.2014.10.043 
Junshan, Z. (2017). The Structure and Management of Administrative Organizations in Universities under the Construction of" Double First-rate". The Science Education Article Collects, (9), 57. https://bit.ly/2YyHu7d

Meyer, J. P., \& Allen. N. J. (1991). A three-component conceptualization of organizational commitment. Human Resource Management Review, 1(1):61-89. https://doi.org/10.1016/1053-4822(91)90011-Z

Osorio-Jaramillo, L. D. R., Ramos-Díaz, E. J., \& Walteros-Astaiza, D. P. (2016). Valores Personales y Compromiso Organizacional: más que una simple relación. Revista de psicología y ciencias del comportamiento de la Unidad Académica de Ciencias Jurídicas y Sociales, 7(2), 33-51. https://bit.ly/3iUn9Ru

Ocando-Cardozo, H. Y. (2017). La supervisión educativa como elemento clave para alcanzar la calidad educativa en las escuelas públicas. Omnia, 23(3), 42-57. https://bit.ly/2FCTYUt

Pacheco-Granados, R. J., Robles-Algarín, C. A., \& Ospino-Castro, A. J. (2018). Análisis de la gestión administrativa en las instituciones educativas de los niveles de básica y media en las zonas rurales de Santa Marta, Colombia. Información tecnológica, 29(5), 259-266. https://bit.ly/34kfqIb

Peña-Cárdenas, M. C., Díaz-Díaz, M., Chávez-Macías, A. G., \& Sánchez-Esparza, C. E. (2016). El compromiso organizacional como parte del comportamiento de los trabajadores de las pequeñas empresas. Revista Internacional Administración \& Finanzas, 9(5), 95-105. https://bit.ly/3h9SK0J

Valaei, N., \& Rezaei, S. (2016). Job satisfaction and organizational commitment: an empirical investigation among ICT-SMEs. Management Research Review, 39(12), 1663-1694. doi: 10.1108/MRR-09-2015-0216

Yousef, D. A. (2017). Organizational commitment, job satisfaction and attitudes toward organizational change: A study in the local government. International Journal of Public Administration, 40(1), 77-88. https://doi.org/10.1080/01900692.2015.1072217 